\title{
Analysis of Influences Considering Vagueness in Agent-Based Simulations
}

\author{
Nobuhide Nakano \\ Faculty of Management, Otemon Gakuin University \\ 2-1-15 Nishi-Ai, Ibaraki, Osaka 567-8502, Japan \\ Tel: +81-72-641-9530, Fax: +81-72-643-9432 \\ E-mail: nakano@res.otemon.ac.jp
}

\begin{abstract}
It is supposed that the elements in circulation of rumors mainly consist of importance and vagueness in psychology and marketing fields. In this paper, the influences of vagueness included in circulation of rumors are modeled and analyzed. Rumors including vagueness element are modeled by local influence in agent-based simulations and agents with vague characters are represented by probability. Simulation studies show that the agents with vagueness perform with some randomness and that the action results of these agents have some regularity.
\end{abstract}

\section{Introduction}

Recently, agent-based simulation problems are attracting much attention in one method for analyzing not only various social and economic phenomena but also marketing research. Agent-based simulation is the method for analyzing various phenomena in virtual spaces which researchers set up. Some of scientists perform their researches using agent-based simulations [1]-[6].

In marketing researches, circulation of rumors has been concentrating much attention of many researchers because many people decide their behaviors by the rumors, for example selections of goods which consumers purchase and choices of a candidate at elections. About the circulation of rumors, Allport and Postman [7] propose the amount of rumors in circulation is proportional to the product of the importance for the rumor and the vagueness of the rumor.

In this paper, the effect of vagueness included rumors is modeled and analyzed by agent-based simulations. In past researches of the author [1], two types of agent, pioneer and follower, are defined and they performed according to the performance rules which are decided by the numbers of neighbors adopting a rumor. In this study, for the decision of attitudes, we introduce the adopting coefficient decided by both the number of neighbors and the vagueness of the rumor. The performance rules of agents are modeled by the adopting coefficient. After simulation studies are performed for various types of agents, the results of analysis are shown.

\section{Proposed Model}

\subsection{Modeling of Agents and Virtual Space}

Rogers [8] categorizes agents at five segments. In the author's study, to simplify the model of agents, two types of agents, pioneers and followers, are employed [1], [2], [6]. "Pioneers" are agents which feel less resistance for employing new objects, that is to say, reformists. On the other hands, "Followers" are agents which feel much resistance for employing new objects, they also called conservative people. Each agent shows an attitude either "adopt" or "reject" for a rumor (a new object).

100 agents (pioneers and/or followers) are arranged in a grid (in a virtual space) with 10-row and 10-column. Each agent decide their attitude (either adoption or rejection) at every time step. Figure 1 illustrates the example of arranged agents in the same virtual space. The left side of Figure 1 shows the pioneers and followers in a virtual space and the right side of Figure 1 shows the adopters and rejecters in the same virtual space as the left 


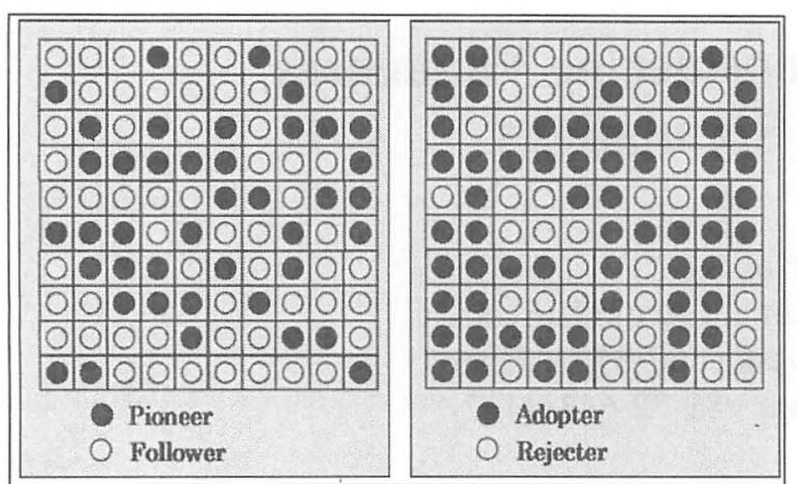

Figure 1. Agents in a virtual space

side of Figure 1. For instance, the agent at the left-bottom corner is a pioneer and his attitude is adoption in Figure 1.

\subsection{Vagueness of Rumors}

Agents decide their attitudes (adoption or rejection) based on the attitudes of their neighbor agents, this rule is called local influence (LI). That is to say, local influence is the effect of rumors which is spread by word of mouth. In past researches [1],[2], local influence was only obtained by attitudes of four agents which exist around the agent which decides an attitude (see Figure 2).

About the circulation of rumors, Allport and Postman [7] propose the fundamental law of rumors shown in equation (1):

$$
\text { (Rumor) } \sim(\text { Importance }) \times(\text { Vagueness }) \text {. }
$$

Equation (1) shows that circulating volume of rumor is proportional to the product of the importance for the rumor and the vagueness of the rumor. In this research, the concept of "Vagueness" in equation (1) is combined with action rule of agents (local influences) in agent-based simulations.

\subsection{Adopting Coefficient}

In order to represent the vagueness of rumors in agent-based simulations, we define the adopting coefficient $\left(=: A_{C}\right)$ as follows:

$$
A_{C}=a \cdot v,
$$

where $a$ is the number of adopters and $v$ is the

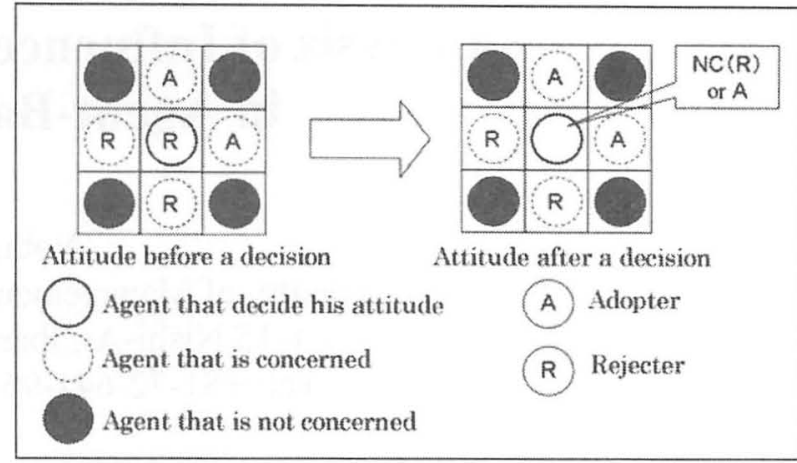

Figure 2. The decision process by local influence

vagueness coefficient. Table 1 shows the action rules of agents (followers and pioneers). In this study we change vagueness coefficient $v$ at random into some ranges based on these action rules.

Table 2 shows the action rules of followers with vagueness, where Attitudes, Reject, Not Change and Adopt are omitted Atti., R, NC and A, respectively. We know that followers sometimes show the different attitudes for the same $a$ at random.

\begin{tabular}{|c|c|c|}
\hline \multirow{2}{*}{$A_{C}$} & \multicolumn{2}{|c|}{ Attitudes } \\
\cline { 2 - 3 } & Pioneer & Follower \\
\hline \hline 0 or more $\sim$ under 1 & Reject & Reject \\
\hline 1 or more $\sim$ under 2 & Adopt & Reject \\
\hline 2 or more $\sim$ under 3 & Not Change & Not Change \\
\hline 3 or more $\sim$ under 4 & Reject & Adopt \\
\hline 4 or more $~$ & Adopt & Adopt \\
\hline
\end{tabular}

Table 1. Action rules of pioneers and followers

Figure 2 shows the decision process of an agent. In this figure, we suppose that the center agent (depicted by solid line) is a follower with adoption, and that his decision rule is the simulation type $S_{1-2}$ shown by Table 2. At first, the number of adopters in neighbor four agents (depicted by broken line) are counted. Black circles are agents which do not concerned with the decision of the center agent. In this figure, as there are two adopters around the center agent, then $A_{C}$ becomes 2 or more and under 4 . Hence, he does not change his attitudes ("reject") or changes his attitude from "reject" to "adopt". The attitude either "reject" or "adopt" is selected at random. 


\begin{tabular}{|c|c|c|c|c|c|c|c|c|c|c|}
\hline $\begin{array}{c}\text { Types of } \\
\text { Simulations. }\end{array}$ & \multicolumn{2}{|c|}{$S_{0-1}$} & \multicolumn{2}{|c|}{$S_{0.5-1}$} & \multicolumn{2}{|c|}{$S_{1 \pm 0.1}$} & \multicolumn{2}{|c|}{$S_{1-2}$} & \multicolumn{2}{|c|}{$S_{1-3}$} \\
\hline Ranges of $v$ & \multicolumn{2}{|c|}{$0 \sim 1$} & \multicolumn{2}{|c|}{$0.5 \sim 1$} & \multicolumn{2}{|c|}{$1 \pm 0.1$} & \multicolumn{2}{|c|}{$1 \sim 2$} & \multicolumn{2}{|c|}{$1 \sim 3$} \\
\hline Adopters $(a)$ & $A_{C}$ & Atti. & $A_{C}$ & Atti. & $A_{C}$ & Atti. & $A_{C}$ & Atti. & $A_{C}$ & Atti. \\
\hline \hline 0 & 0 & $\mathrm{R}$ & 0 & $\mathrm{R}$ & 0 & $\mathrm{R}$ & 0 & $\mathrm{R}$ & 0 & $\mathrm{R}$ \\
\hline 1 & $0 \sim 1$ & $\mathrm{R}$ & $0.5 \sim 1$ & $\mathrm{R}$ & $0.9 \sim 1.1$ & $\mathrm{R}$ & $1 \sim 2$ & $\mathrm{R}$ & $1 \sim 3$ & $\begin{array}{c}\mathrm{R} \\
\mathrm{NC}\end{array}$ \\
\hline 2 & $0 \sim 2$ & $\mathrm{R}$ & $1 \sim 2$ & $\mathrm{R}$ & $1.8 \sim 2.2$ & $\begin{array}{c}\mathrm{R} \\
\mathrm{NC}\end{array}$ & $2 \sim 4$ & $\begin{array}{c}\mathrm{NC} \\
\mathrm{A}\end{array}$ & $2 \sim 6$ & $\begin{array}{c}\mathrm{NC} \\
\mathrm{A}\end{array}$ \\
\hline 3 & $0 \sim 3$ & $\begin{array}{c}\mathrm{R} \\
\mathrm{NC}\end{array}$ & $1.5 \sim 3$ & $\begin{array}{c}\mathrm{R} \\
\mathrm{NC}\end{array}$ & $2.7 \sim 3.3$ & $\begin{array}{c}\mathrm{NC} \\
\mathrm{A}\end{array}$ & $3 \sim 6$ & $\mathrm{~A}$ & $3 \sim 9$ & $\mathrm{~A}$ \\
\hline 4 & $0 \sim 4$ & $\begin{array}{c}\mathrm{R} \\
\mathrm{NC} \\
\mathrm{A}\end{array}$ & $2 \sim 4$ & $\mathrm{NC}$ & $3.6 \sim 4.4$ & $\mathrm{~A}$ & $4 \sim 8$ & $\mathrm{~A}$ & $4 \sim 12$ & $\mathrm{~A}$ \\
\hline
\end{tabular}

Table 2. Action rules of followers with vagueness

\section{Simulation Method}

Recalling the mentioned above, the procedure for the simulation is performed by the following steps:

[1] 100 agents are placed in a grid (10-row and 10 -colums) and the number of initial pioneers $\left(=: P_{I N I}\right)$, the number of initial adopters $\left(=: A_{I N I}\right)$ and vagueness coefficient $v$ of followers are set. Initial arrangements of agent and initial attitudes (adoption or rejection) are both random and have no connection.

[2] Each agent decides own attitude once every one time step. The attitude of each agent at $(t+1)$-th time step is decided by calculating $A_{C}$ at $t$-th time step.

[3] A trial defined as a simulation performed at 100 time step in step [2] is performed.

[4] To evaluate results of simulations, the selected average adopters calculated by last 10 time steps (data of from 91st time step to 100th time step) is defined as the final average adopters of the trial (see Figure 3).

[5] As the initial arrangements of agents and initial attitudes are random, the results of simulations become different in spite of a same initial condi$\operatorname{tion}\left(P_{I N I}\right.$ and $\left.A_{I N I}\right)$. Then 1000 trials are performed at the same initial condition (only initial arrangements of pioneers and/or followers are different).

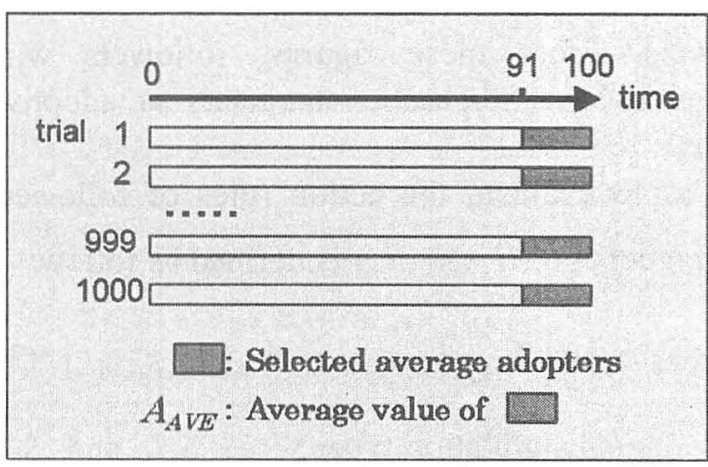

Figure 3. Method of simulations for one set of initial conditions

[6] Then the average value of these final average adopters $\left(=: A_{A V E}\right)$ is calculated (see Figure 3).

[7] $A_{A V E}$ is calculated by changing $P_{I N I}$ and $A_{I N I}$ from 0 to 100 , respectively.

\section{Results and Analysis}

Before showing simulation results, we define three types of agents to compare with the simulations with vagueness. In this study, we set $v=1$ at pioneers, then the action rules of followers are only changed according to $v$. Table 3 shows action rules of followers at the simulations without vagueness. The results for simulation studies of simulation types $S_{0-1}, S_{0.5-1}, S_{1 \pm 0.1}, S_{1-2}, S_{1-3}$, 


\begin{tabular}{|c|c|c|c|c|c|c|}
\hline $\begin{array}{c}\text { Simula- } \\
\text { tion types }\end{array}$ & \multicolumn{2}{|c|}{$S_{0.8}$} & \multicolumn{2}{c|}{$S_{1}$} & \multicolumn{2}{c|}{$S_{2}$} \\
\hline$v$ & \multicolumn{2}{|c|}{0.8} & \multicolumn{2}{|c|}{1} & \multicolumn{2}{|c|}{2} \\
\hline $\begin{array}{c}\text { Adopters } \\
(a)\end{array}$ & $A_{C}$ & Atti. & $A_{C}$ & Atti. & $A_{C}$ & Atti. \\
\hline 0 & 0 & $\mathrm{R}$ & 0 & $\mathrm{R}$ & 0 & $\mathrm{R}$ \\
\hline 1 & 0.8 & $\mathrm{R}$ & 1 & $\mathrm{R}$ & 2 & $\mathrm{NC}$ \\
\hline 2 & 1.6 & $\mathrm{R}$ & 2 & $\mathrm{NC}$ & 4 & $\mathrm{~A}$ \\
\hline 3 & 2.4 & $\mathrm{NC}$ & 3 & $\mathrm{~A}$ & 6 & $\mathrm{~A}$ \\
\hline 4 & 3.2 & $\mathrm{~A}$ & 4 & $\mathrm{~A}$ & 8 & $\mathrm{~A}$ \\
\hline
\end{tabular}

Table 3. Action rules of followers without vagueness

$S_{0.8}, S_{1}$ and $S_{2}$ illustrate in Figures 4, 5, 6, 7, 8, 9,10 and 11 , respectively. Figure 12 is depicted the trajectory at $P_{I N I}=0$, where all agents are only followers. From these figures, followers with vagueness have stochastic characters at adoptive actions.

So as to evaluate the action rules of followers with vagueness, $d_{s}\left(S_{b}, S_{c}\right)$ is defined as follow:

$$
d_{S}\left(S_{b}, S_{c}\right)=\left|A_{A V E}\right|_{S_{b}, P_{N I}=0}-\left.A_{A V E}\right|_{S_{c}, P_{N I}=0} \mid \text {. }
$$

We evaluated simulation types $S_{1 \pm 0.1}, S_{1-2}$ and $S_{1-3}$ by comparing with followers without vagueness, whose results illustrate in Figures 13, 14 and 15, respectively. We know that the character $S_{1 \pm 0.1}$ is intermediate between $S_{0.8}$ and $S_{1}$. Furthermore, we see that the character of $S_{1-2}$ is partial towards $S_{1}$ rather than $S_{2}$ and that the character of $S_{1-3}$ is partial towards $S_{2}$ rather than $S_{1}$. We see that the degree of partialness on characters can be represented by adopting coefficients with vagueness.

\section{Conclusions}

In this paper, we complex the concept of vagueness for rumors into characters of agents in agent-based simulations. The points are that the adopting actions of agents with vagueness show random attitudes and that the partialness in agents with vagueness can be described by random element. Concretely speaking, the degree of partialness in characters can be described by stochastic process. Though the relation (1) is simple, we also can show this theory corresponds to reality (the adopting actions of persons) by this study.

Though the author shows the ability of agent-based simulations with probability until now, precisely analyses for stochastic characters in agent-based simulations are needed for the next step.

\section{References}

[1] N. Nakano: An Agent-Based Approach to Simulations of Global Influence with Random Effect, Trans. International Journal of Innovative Computing, Information and Control, Vol. 2, No. 5, pp.1153-1163, 2006.

[2] N. Nakano: A Pilot Study on Modeling and Analysis of Capricious Agents in Agent-Based Simulations, Trans. International Journal of Innovative Computing, Information and Control, Vol. 4, No.1, pp.91-100, 2008.

[3] M. Paolucci and R. Sacile: Agent-Based Manufacturing and Control Systems, CRC Press, Florida, 2005.

[4] J. M. Epstein and R. Axtell: Growing Artificial Societies ---Social Science from the Bottom Up---, the Brookings Institution., N.Y., 1996.

[5] M. Aoyagi and A. Namatame: Massive Individual Based Simulation: Forming and Reforming of Agents Flocking, Proc. 35th SICE Regional Conference at System Engineering Technical Committee --- Creation of Agent Based Social System Science ---, pp.135-140, 2005.

[6] T. Murata and T. Yagi: The Relation Between Distribution of Initial Adoptions and Propagation of Adoptive Actions in Agent-Based Simulations, Proc. 48th Annual Conference of the ISCIE, pp.177-178, 2004 (in Japanese).

[7] G. W. Allport and L. Postman: The Psychology of Rumor, Iwanami Gendai Sōsho, 1952 (translated by H. Minami, in Japanese).

[8] E. M. Rogers: Diffusion of Innovations (Third Edition), the Free Press., N.Y., 1983. 


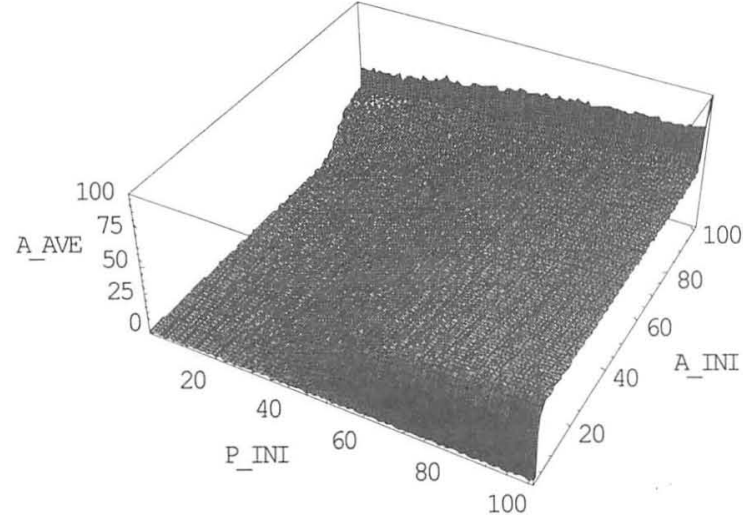

Figure 4. $A_{A V E}$ at the simulation type $S_{0-1}$

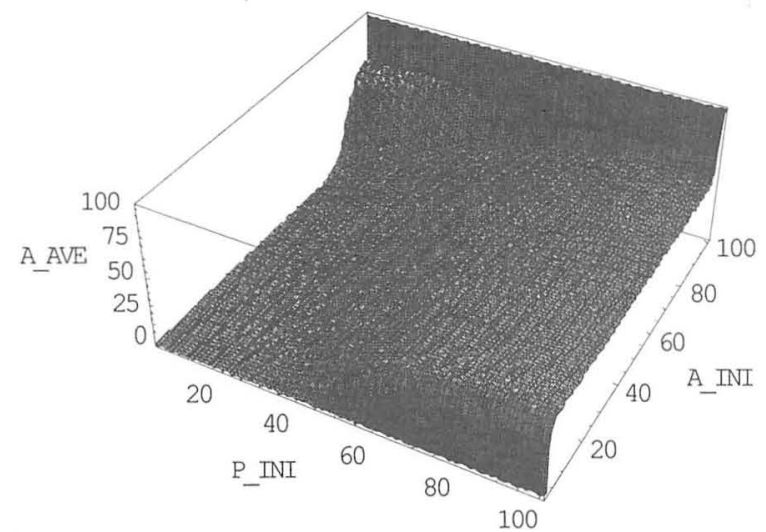

Figure 5. $A_{A V E}$ at the simulation type $S_{0.5-1}$

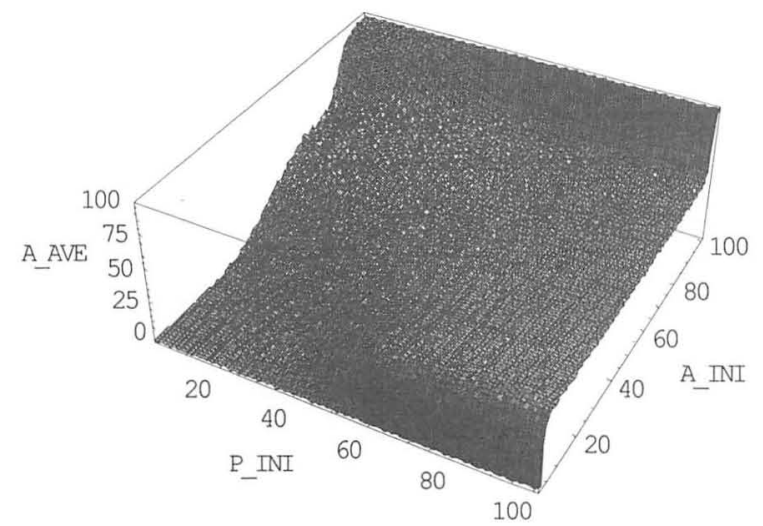

Figure 6. $A_{A V E}$ at the simulation type $S_{1 \pm 0.1}$

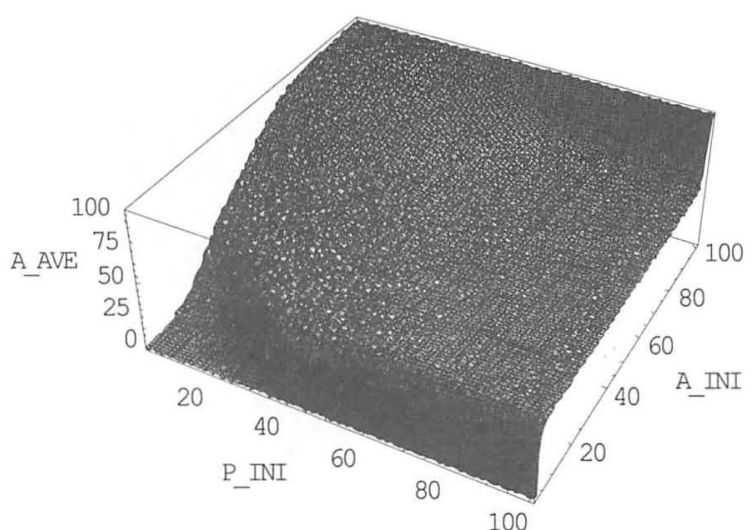

Figure 7. $A_{A V E}$ at the simulation type $S_{1-2}$

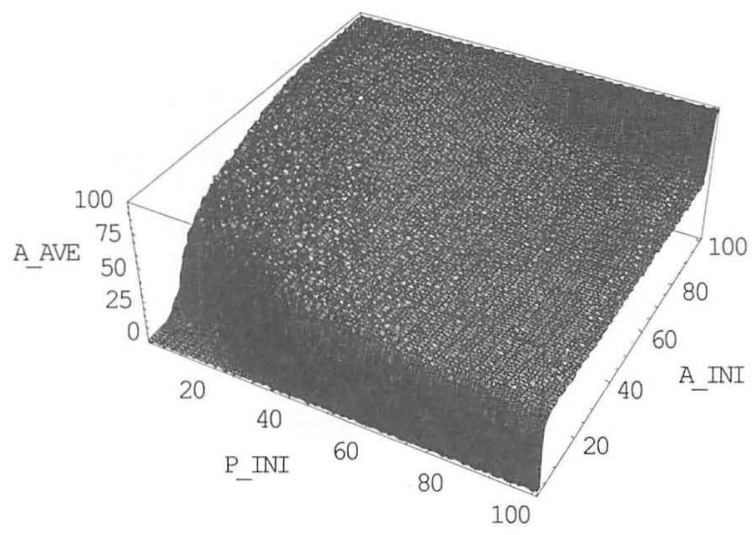

Figure 8. $A_{A V E}$ at the simulation type $S_{1-3}$

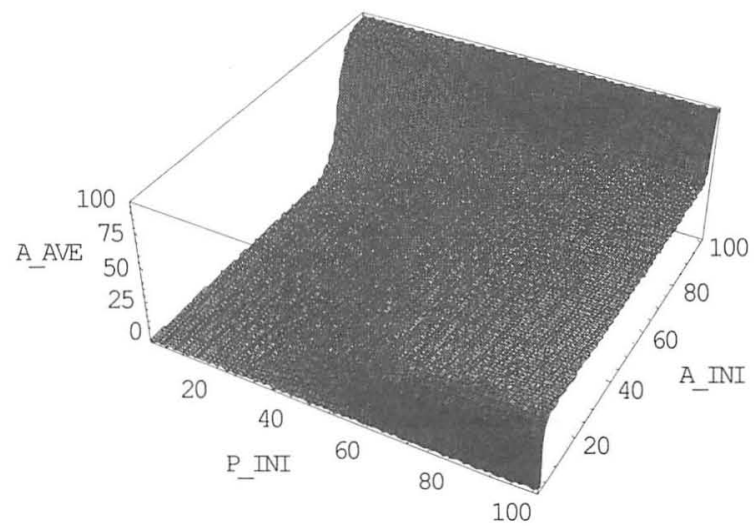

Figure 9. $A_{A V E}$ at the simulation type $S_{0.8}$ 


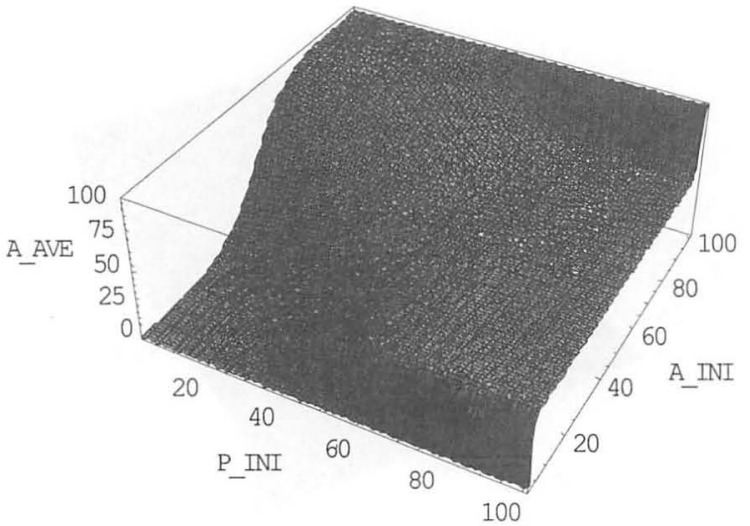

Figure 10. $A_{A V E}$ at the simulation type $S_{1}$

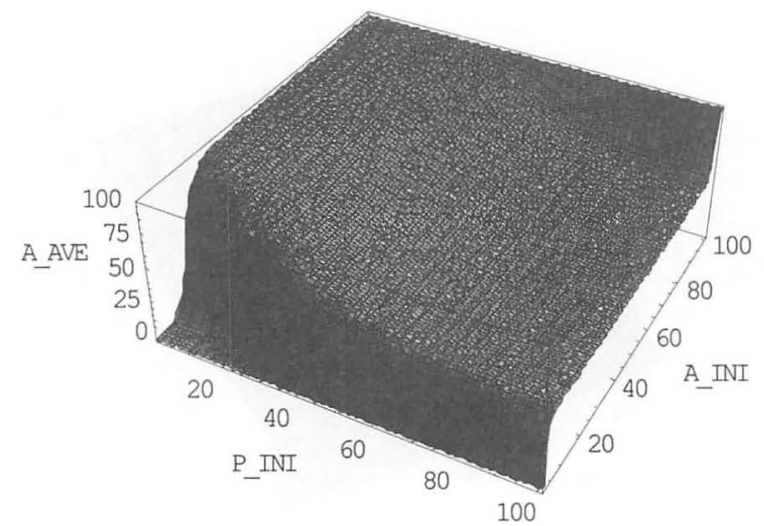

Figure 11. $A_{A V E}$ at the simulation type $S_{2}$

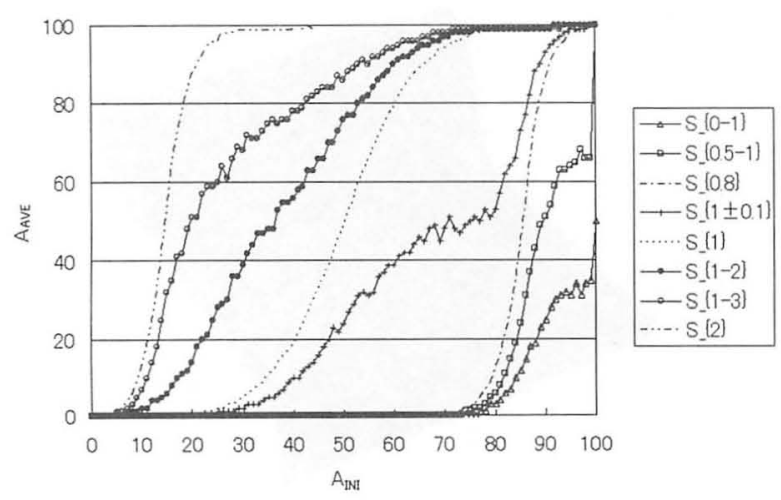

Figure 12. $A_{A V E}$ at $P_{I N I}=0$

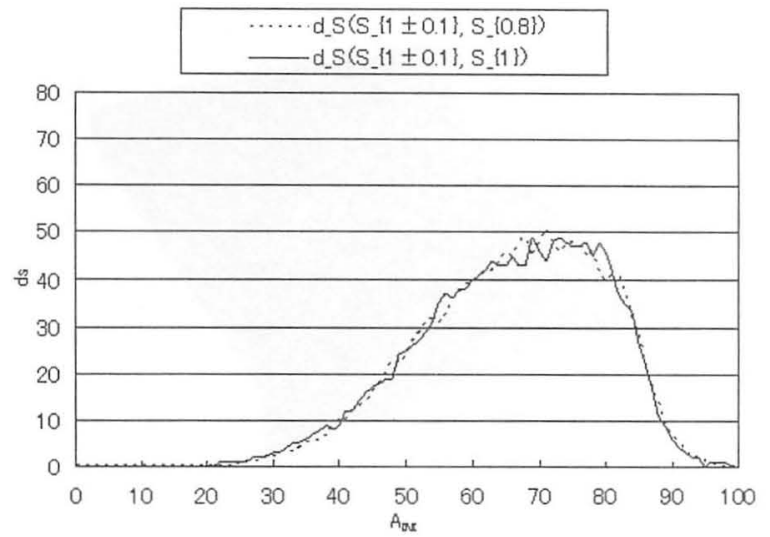

Figure 13. $d_{S}\left(S_{1 \pm 0.1}, S_{0.8}\right)$ and $d_{S}\left(S_{1 \pm 0.1}, S_{1}\right)$

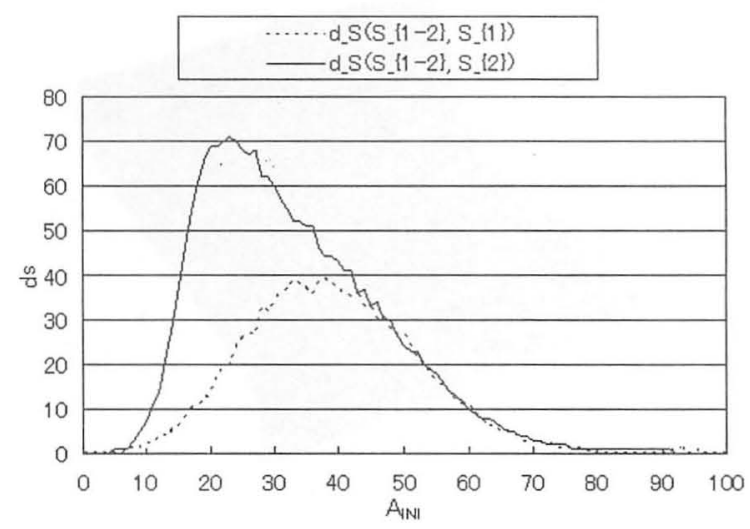

Figure 14. $d_{S}\left(S_{1-2}, S_{1}\right)$ and $d_{S}\left(S_{1-2}, S_{2}\right)$

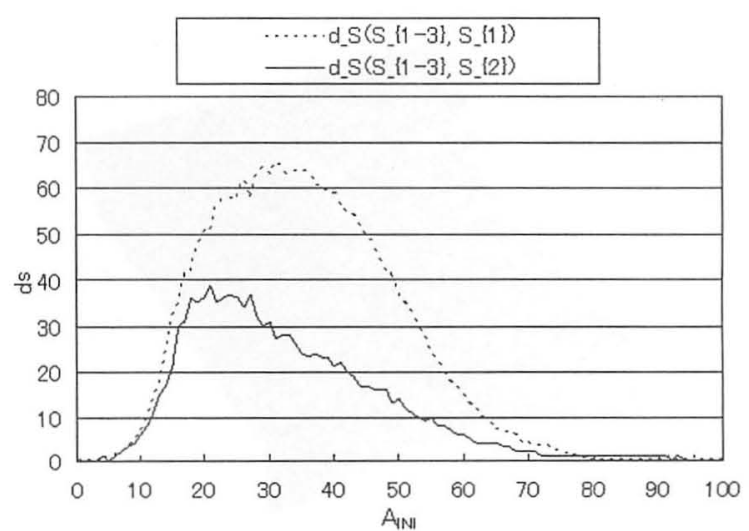

Figure 15. $d_{S}\left(S_{1-3}, S_{1}\right)$ and $d_{S}\left(S_{1-3}, S_{2}\right)$ 